\title{
A randomized trial comparing inspiratory training and positive pressure training in immediate lung recovery after minor pleuro-pulmonary surgery
}

\author{
Sabina Isabel Lähteenmäki ${ }^{1,2^{\wedge}}$, Thanos Sioris ${ }^{2}$, Heidi S. S. Mahrberg ${ }^{2}$, Irina C. Rinta-Kiikka ${ }^{3}$, \\ Jari O. Laurikka,
}

${ }^{1}$ Faculty of Medicine and Health Technology, Tampere University, Tampere, Finland; ${ }^{2}$ Tampere Heart Hospital, Tampere, Finland; ${ }^{3}$ Imaging Centre, Department of Radiology, Tampere University Hospital, Tampere, Finland

Contributions: (I) Conception and design: JO Laurikka, HSS Mahrberg, IC Rinta-Kiikka; (II) Administrative support: JO Laurikka, IC Rinta-Kiikka, T Sioris; (III) Provision of study materials or patients: All authors; (IV) Collection and assembly of data: JO Laurikka, HSS Mahrberg, IC Rinta-Kiikka, SI Lähteenmäki; (V) Data analysis and interpretation: All authors; (VI) Manuscript writing: All authors; (VII) Final approval of manuscript: All authors. Correspondence to: Sabina Isabel Lähteenmäki. Faculty of Medicine and Health Technology, Tampere University, Tampere, Finland.

Email: sabina.lahteenmaki@tuni.fi.

\begin{abstract}
Background: Two respiratory physiotherapy modalities were compared in a randomized controlled trial on patients undergoing minor pleuro-pulmonary surgery.

Methods: Forty-five patients were randomly allocated into positive expiratory pressure (PEP) therapy $(n=23)$ and inspiratory muscle training (IMT) groups $(n=22)$. Individualized group specific physiotherapeutic guidance was administered preoperatively, and once a day postoperatively. Patients also performed independent exercises and kept a logbook. Pain was assessed on a numerical reference scale (NRS). Volumetric pulmonary function values and walking distance were recorded preoperatively, and on first (POD1) and second postoperative days (POD2). Pre- and postoperative values were compared using two-way repeated measures analysis of variance.
\end{abstract}

Results: Patient characteristics and pleuro-pulmonary interventions were similar between the groups. Thoracotomy was performed in 14/45 and video assisted surgery (VATS) in 31/45 of cases. Preoperative volumetric pulmonary functions were normal or slightly decreased in $29 / 45$, and fell significantly $(\mathrm{P}<0.001)$ on the first postoperative day (POD1) and improved but remained significantly lower on the second postoperative day. The recovery of mean FEV1, FIV1 and FIVC values was greater in the IMT than in the PEP group between POD1 and POD2, but without significant difference. The corresponding relative to preoperative values were higher in the IMT group, with a significant difference in FEV1 ( $\mathrm{P}=0.045)$. Also relative PEF and FIV1 values seemed to be slightly higher in the IMT compared to the PEP group, but not significantly. Average NRS values for pain were lower in the IMT group $(\mathrm{P}=0.010)$ but only on POD1. Air leak was noted in 4/45 patients, two in each group, on POD1, and two in PEP groups and one in IMT group on POD2. Mean measured walking distances between groups did not differ. Mean hospital stay was 4 days in the PEP group and 3 days in the IMT group. There was no hospital mortality.

Conclusions: Pulmonary function values decreased significantly after minor lung resections, supporting rehabilitative respiratory physiotherapy to avoid postoperative pulmonary complications (PPCs). Both PEP and IMT training were well tolerated and equally efficient when comparing spirometry values at three time points. IMT appeared advantageous regarding relative FEV1 recovery and immediate postoperative pain.

Keywords: Minor lung resection; pulmonary function; respiratory physiotherapy; positive expiratory pressure training (PEP training); inspiratory muscle training (IMT)

^ ORCID: 0000-0003-4308-0170. 
Submitted Jan 11, 2021. Accepted for publication Jun 21, 2021.

doi: $10.21037 /$ jtd-21-473

View this article at: https://dx.doi.org/10.21037/jtd-21-473

\section{Introduction}

The speed of postoperative lung recovery after thoracic surgery significantly affects patients' overall recovery time and costs of hospitalization. Impaired respiration predisposes to postoperative pulmonary complications (PPCs) which may prolong hospitalization and increase its costs (1-3). Recovery is promoted by respiratory physiotherapy, it's benefits having been demonstrated after CABG surgery (4) and major lung resections (5-19). Preand postoperative rehabilitation in lung cancer surgery is advised by European Respiratory Society/European Society of Thoracic Surgeons (ERS/ESTS) guidelines (20).

Several studies have demonstrated the benefits of inspiratory muscle training (IMT) for pre- and postoperative rehabilitation as a decreased incidence of PPCs and shorter hospitalization (14,21-24). IMT has also improved the results of six-minute walking tests of lung transplant candidates (25) and the exercise tolerance of chronic obstructive pulmonary disease (COPD) patients (26). Our previous study demonstrated IMT being as efficient as water bottle positive expiratory pressure (PEP) training in major lung resection patients, and as a more feasible form of physiotherapy in case of postoperative air leak (27).

The majority of previous studies has involved patients undergoing major lung resection or with COPD as a significant comorbidity. In patients undergoing minor, less than lobectomy lung resections, the permanent amount of functioning lung tissue is only marginally reduced. Evidence concerning the need for respiratory rehabilitation in patients undergoing minor lung resections is limited. Many patients have a benign diagnosis and fare well. However, significant complications and hospital mortality are reported because of the underlying lung disease. This is especially the case in patients with advanced lung fibrosis, disseminated primary or secondary pulmonary or pleural malignancy (28). The life expectancy of these patients is severely limited, so complications and prolonged hospitalization after surgery should be prevented at all costs also for compassionate reasons.

The aim of this prospective randomized study was to determine if IMT respiratory physiotherapy could improve immediate postoperative recovery of respiratory function better than conventional water bottle PEP in patients undergoing minor lung resections. We present the following article in accordance with the CONSORT reporting checklist (available at https://dx.doi.org/10.21037/ jtd-21-473).

\section{Methods}

Our study was made of 45 patients who underwent lung biopsies and other minor thoracic operations at Tampere University Heart Hospital, Finland, between May 2013 and February 2016 (Figure 1). Patients unable to co-operate because of a psychiatric condition, neurological ailment severely affecting respiration, intoxication upon admission, infective pulmonary disease or contagious infection including tuberculosis, recent or acute febrile respiratory infection, preoperative cardiac pacemaker, severe respiratory failure $\left(\mathrm{SpO}_{2}<90\right.$ or $\mathrm{pO}_{2}<8$ or respiratory rate at rest over $25 / \mathrm{min}$ or night-time oxygen therapy) or legally incapacitated patients were excluded from the study. Participants were enrolled by the investigators (JL and $\mathrm{HM}$ ) and were randomly allocated to two treatment groups after signing the informed consent files. Recruiting was terminated upon enrolment of the predetermined number of patients.

Patients were randomized 1:1 to parallel open label groups receiving conventional respiratory physiotherapy (PEP, 23 subjects) or inspiratory muscle training (IMT, 22 subjects) prior to surgery by using randomization procedure (computerized random numbers in sealed envelopes). The groups were compared according to intention to treat. The surgical procedures included lung and pleural biopsies, tumor excisions, wedge resections of the lung by VATS or thoracotomy.

No deviations from intended therapy or dropping out from the assigned groups occurred. The study protocol (R13037) was approved by the Ethics Committee of the Pirkanmaa Hospital District, Tampere, Finland, and was conducted in accordance with the Declaration of Helsinki (as revised in 2013). Written informed consent was obtained from all patients before starting the investigations. Our study protocol is filed at ClinicalTrials.gov.

One physiotherapist (HM) instructed the assigned 


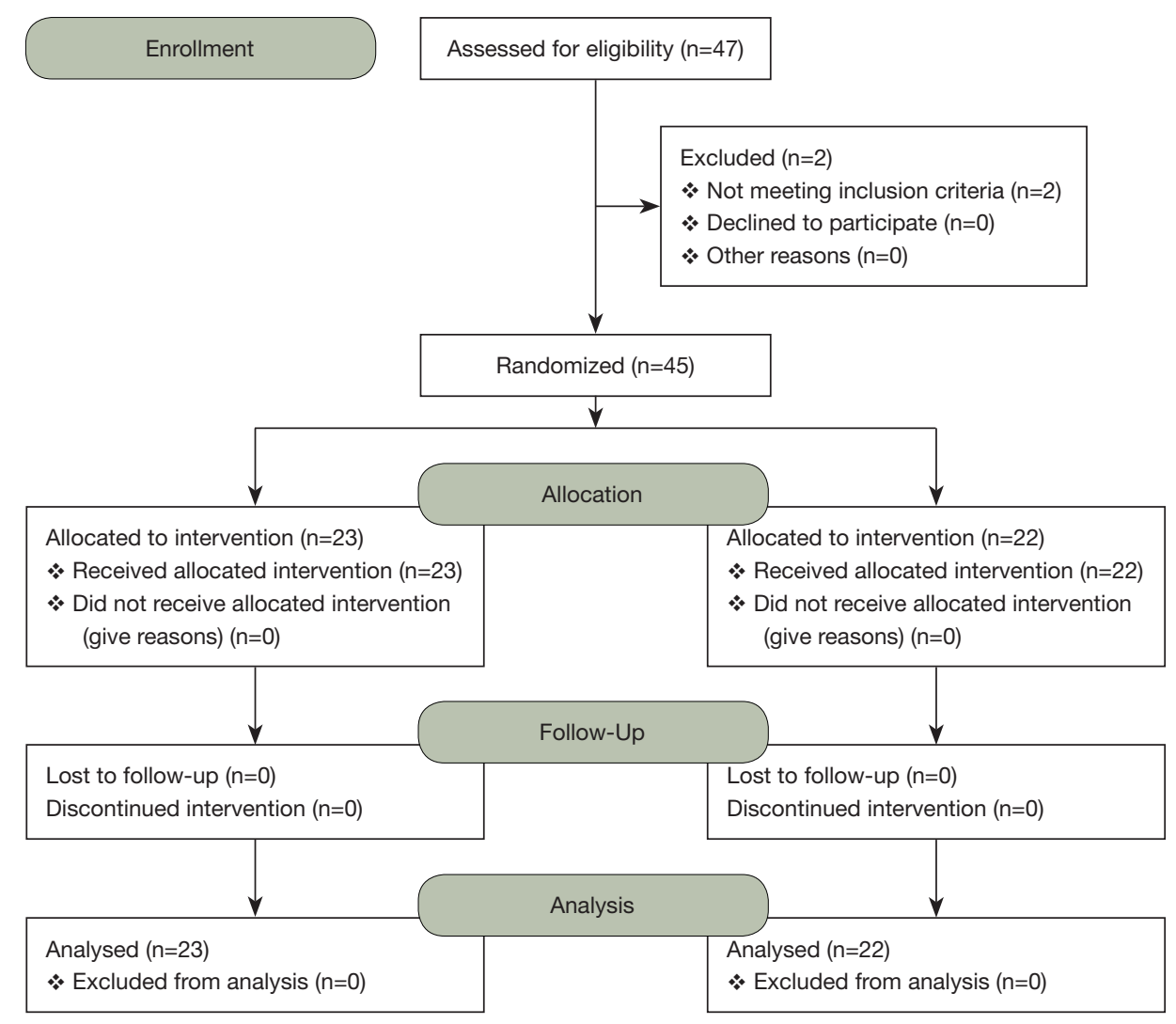

Figure 1 Participant flow chart.

physiotherapy (PEP or IMT) to each patient on the preoperative day, and on the first and second postoperative day. Exercises were performed once a day with the physiotherapist, and the patients were encouraged to do independent exercises at least five times a day. In the water bottle PEP group, a basic pressure of $10 \mathrm{cmH}_{2} \mathrm{O}$ was used. For IMT physiotherapy, a ThresholdIMT (Philips Respironics, Murrysville, Pennsylvania, United States) apparatus was employed. Pressure was set at $20 \%$ of the individuals' preoperative maximal inspiratory pressure (MIP). A Thopaz automated pleural suction device (Medela AG, Baar, Switzerland) was connected to the chest tubes in all the patients. The same physiotherapist assigned any observed post-operative air leak onto three categories based on the readout of the Thopaz ( $0=$ no air leakage, $1=$ low grade (under $200 \mathrm{~mL}$ ), $2=$ moderate or profuse (200 $\mathrm{mL}$ or above). The expiratory resistance of the PEP water bottle was lowered to $5-7 \mathrm{cmH}_{2} \mathrm{O}$ if air leak rose during physiotherapy to $200 \mathrm{~mL}$ or higher, or if a lesser increase was observed on repeated occasions.

Additionally, patients were guided to walk a minimum of 3-4 rounds on POD1 and at least five rounds on POD2, and to record respiratory exercises and walks on a logbook. They were also assigned routine exercises including shoulder mobilization and manual support of the incision site when coughing. Intensity of pain was measured using a numeric scale (NRS) from 0 to 10.

Pulmonary function tests were conducted on all three days of the study: preoperatively before allocation, and on POD1 and POD2 after performing the physiotherapeutic exercises. For 22 patients in PEP group and 22 in IMT group, full pulmonary function test results for the three study days were obtained. The Medikro Pro (Medikro Oy, Kuopio, Finland) portable spirometry device was used for bedside pulmonary function testing in all patients. The device is connectable to a portable computer, and the software includes reference values for pulmonary functions of the Finnish general population (29). Pre-operative pulmonary functions were defined as normal, mildly decreased, moderately decreased or severely decreased in accordance to these reference values (Table 1).

The same radiologist (IRK) evaluated the images from 
Table 1 Main patient parameters in the study groups

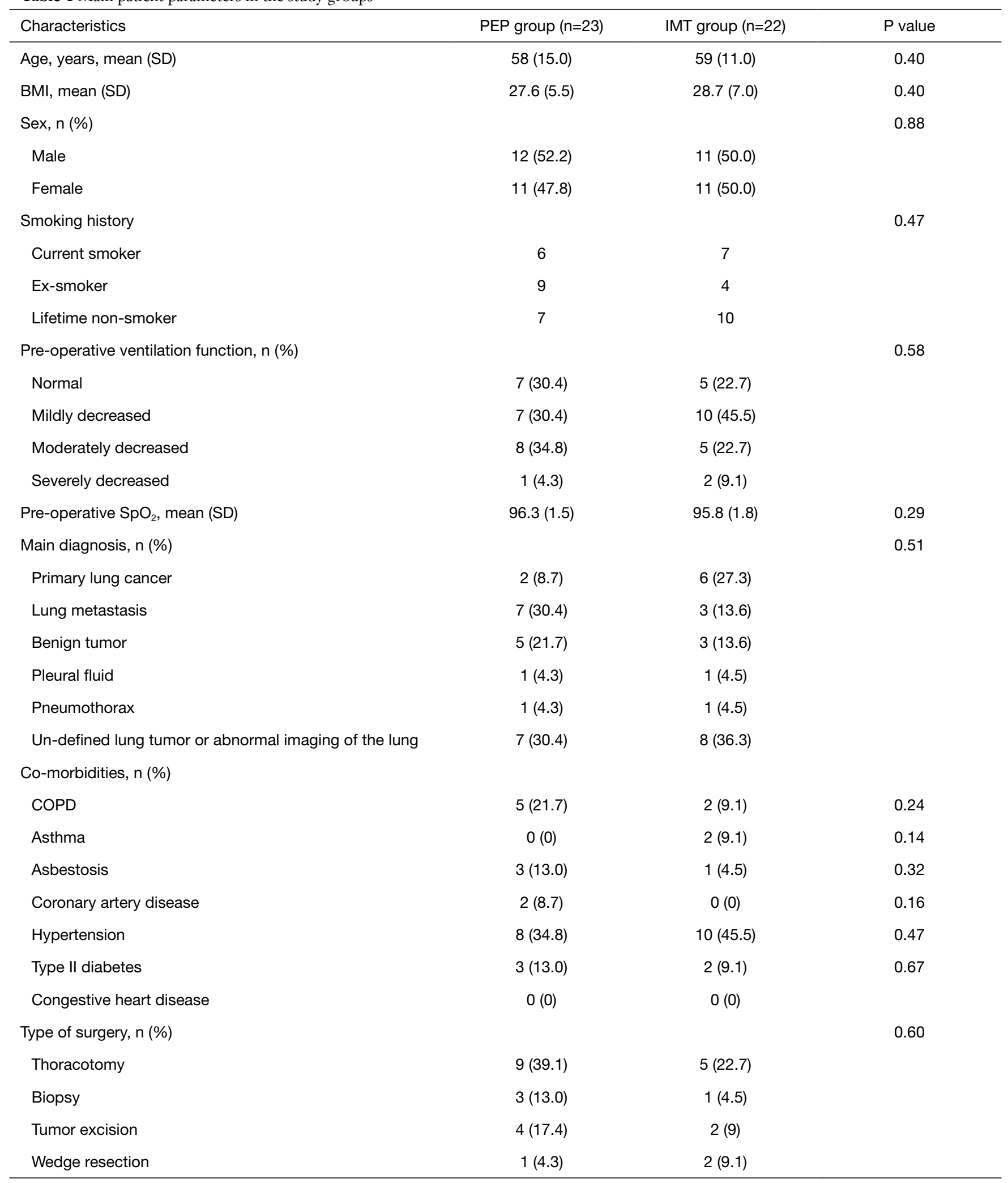

Table 1 (continued) 
Table 1 (continued)

\begin{tabular}{lcc}
\hline Characteristics & PEP group $(\mathrm{n}=23)$ & IMT group $(\mathrm{n}=22)$ \\
\hline Pleurectomy & $1(4.3)$ & $0(0)$ \\
VATS, $\mathrm{n}(\%)$ & $14(60.9)$ & $17(77.3)$ \\
Biopsy & $8(34.8)$ & $6(27.3)$ \\
Tumor excision & $3(13.0)$ & $7(31.8)$ \\
Wedge resection & $3(13.0)$ & $4(18.2)$ \\
\hline
\end{tabular}

Current smoker: smoking till date of surgery. Ex-smoker: abstained for minimum of two weeks prior to surgery. Non-smoker: has never smoked. Normal: FEV1 80-126\%; mildly decreased: FEV1 65-79\%; moderately decreased: FEV1 45-64\%; severely decreased FEV1 25-44\%. COPD, chronic obstructive pulmonary disease; VATS, video assisted thoracic surgery; PEP, positive expiratory pressure; IMT, inspiratory muscle training.

all patients, measured and marked the area of the airfilled lung, the areas of atelectasis, the presence and size of pneumothorax, the amount of pleural fluid, and calculated the change in the area of the air-filled lung between preoperative and postoperative images. The volume and change of the air-filled lung were evaluated in both PA- and lateral views. The amount of atelectasis was expressed in three levels $(0=$ no atelectasis, $1=$ atelectasis less than $25 \%$ of lung volume, $2=$ atelectasis $25-50 \%$ of lung volume). Amount of pleural fluid was expressed in four levels $(0=$ no pleural fluid, $1=$ pleural fluid under $250 \mathrm{~mL}, 2=$ pleural fluid 250-500 mL, $3=$ pleural fluid over $500 \mathrm{~mL}$ ). Postoperative pneumothorax was expressed as three levels $(0=$ no pneumothorax, $1=$ small pneumothorax under $2 \mathrm{~cm}$, $2=$ pneumothorax over $2 \mathrm{~cm}$ or wide-range, $3=$ most of the lung collapsed).

Pulmonary function test values, primarily forced expiratory volume during one second (FEV1), as measured with volumetric spirometry, radiological findings and complications affecting e.g., length of hospital stay was assessed as primary outcomes and measures of compliance and patient-reported daily walking distances (meters walked during the last 24 hours) as secondary outcomes.

\section{Statistical methods}

Based on a pilot study, the $80 \%$ power to detect a postoperative change of $0.3 \mathrm{~L} / \mathrm{sec}$ in main parameter FEV1 (with SD 0.3) with type I error level (5\%) was calculated to need 17 patients recruited per group. Statistical comparisons were made with IBM SPSS Statistics for Windows 24 (IBM Corp, Armonk, New York, United States). The normal distribution of numerical values was tested by Shapiro-
Wilk test. In the case of normal distribution, comparison of the mean values of the variables was done with the $t$-test of independent variables. Differences between the classified variables were tested by cross-tabulation and Pearson's $\chi^{2}$ testing.

Differences between intervention groups in spirometry values and walking distances were analyzed using two-way mixed factors repeated measures analysis as a function of time. To examine the appropriateness of the analysis, the normal distribution and possible outliers were assessed, as well as the homogeneity of variances with the Levene test, covariance equivalence with the Box test, and Mauchly's test of sphericity were performed. Differences were considered statistically significant when $\mathrm{P}<0.05$.

\section{Results}

Patient demographics and general data are given in Table 1 . No statistical or clinical differences between the groups in demographics or medical or respiratory conditions were found between the groups. 16 out of 23 PEP group patients and 17 out of 22 IMT group patients had pulmonary function values mildly, moderately or severely decreased. This stands for $70 \%$ vs. $77 \%$, but the difference was not statistically significant. PEP group had more patients with moderately decreased pulmonary functions and IMT groups had more patients with mildly decreased pulmonary functions. Difference between the intervention groups was not statistically significant. Two of the IMT group patients had asthma as a comorbidity, but neither of them used regularly bronchodilatators on corticosteroids on admission or during the study period.

The results of the measured pulmonary function tests are 
Table 2 Pre- and post-operative pulmonary function values in the study groups

\begin{tabular}{|c|c|c|c|c|c|c|c|c|c|}
\hline \multirow[t]{2}{*}{$\begin{array}{l}\text { Variable } \\
\text { (SD) }\end{array}$} & \multicolumn{3}{|c|}{ PEP } & \multirow{2}{*}{$\begin{array}{l}\text { Preop vs. } \\
\text { 1POD, } \\
\text { 1POD vs. } \\
\text { 2POD } \\
\text { P value }\end{array}$} & \multicolumn{3}{|c|}{ IMT } & \multirow{2}{*}{$\begin{array}{c}\text { Preop vs. } \\
\text { 1POD, } \\
\text { 1POD vs. } \\
\text { 2POD } \\
\text { P value }\end{array}$} & \multirow{2}{*}{$\begin{array}{c}\text { Between } \\
\text { groups PEP vs } \\
\text { IMT, repeated } \\
\text { measures } \\
\begin{array}{c}\text { F-statistic } \\
\text { (P value) }\end{array}\end{array}$} \\
\hline & Preop & 1POD & 2POD & & Preop & 1POD & 2POD & & \\
\hline FVC\% & $87.96(24.16)$ & $56.39(18.84)$ & 63.05 (21.10) & $\begin{array}{l}P<0.001 \\
P=0.003\end{array}$ & $81.55(17.18)$ & $59.50(18.70)$ & $66.18(18.85)$ & $\begin{array}{l}P<0.001 \\
P=0.001\end{array}$ & $0.004(0.947)$ \\
\hline FEV1, L/sec & $2.68(0.89)$ & $1.66(0.63)$ & $1.86(0.71)$ & $\begin{array}{l}P<0.001 \\
P=0.023\end{array}$ & $2.50(0.86)$ & $1.79(0.66)$ & $2.03(0.73)$ & $\begin{array}{l}P<0.001 \\
P=0.006\end{array}$ & $0.042(0.838)$ \\
\hline PEF\% & $83.77(32.56)$ & $48.73(17.97)$ & $53.86(19.00)$ & $\begin{array}{l}P<0.001 \\
P=0.020\end{array}$ & $78.77(16.37)$ & $53.91(18.54)$ & $62.77(17.72)$ & $\begin{array}{l}P<0.001 \\
P<0.001\end{array}$ & $0.318(0.576)$ \\
\hline PIF, L/sec & $5.97(1.91)$ & 3.96 (1.52) & $3.96(1.63)$ & $\begin{array}{l}P<0.001 \\
P=0.989\end{array}$ & $5.55(1.88)$ & $3.71(1.38)$ & $4.11(1.77)$ & $\begin{array}{l}P<0.001 \\
P=0.053\end{array}$ & $0.137(0.713)$ \\
\hline PIF\% & $93.05(26.17)$ & $60.45(19.44)$ & $60.36(20.23)$ & $\begin{array}{l}P<0.001 \\
P=0.978\end{array}$ & 87.05 (24.88) & $58.91(20.34)$ & $65.18(25.42)$ & $\begin{array}{l}P<0.001 \\
P=0.066\end{array}$ & $0.024(0.878)$ \\
\hline FIVC, L/sec & 3.67 (1.09) & $2.45(1.00)$ & 2.69 (1.09) & $\begin{array}{l}P<0.001 \\
P=0.019\end{array}$ & $3.42(1.03)$ & $2.48(0.82)$ & $2.79(0.99)$ & $\begin{array}{l}P<0.001 \\
P=0.003\end{array}$ & $0.023(0.880)$ \\
\hline
\end{tabular}

FVC forced vital capacity: the determination of the vital capacity from a maximally forced expiratory effort; L/sec, litres per second; SD, standard deviation; FVC\%, forced vital capacity in proportion to control populations results; FEV1, forced expiratory volume during one second; FEV1\%, forced expiratory volume during one second in proportion to control populations results; PEF, peak expiratory flow; PEF\%, peak expiratory flow in proportion to control populations results; PIF, peak inspiratory flow; PIF\%, peak inspiratory flow in proportion to control populations results; FIVC, forced inspiratory vital capacity; FIV1, forced inspiratory volume during one second; MIP, maximal inspiratory pressure. Preop, values on day before surgery; 1POD, first postoperative day; 2POD, second postoperative day.

given in the Table 2. There was no statistically significant difference in any preoperative spirometry values between intervention groups. The pulmonary function values in FEV1, forced vital capacity (FVC), peak expiratory flow (PEF), forced inspiratory vital capacity (FIVC), forced inspiratory volume in one second (FIV1), and peak inspiratory flow (PIF) all decreased significantly at first and second postoperative day as compared to preoperative value in both study groups. The decrease in FEV1, and of the FEV1 in proportion to control populations results (FEV1\%) was smaller in IMT group than in the PEP group, but without statistical significance (FEV1: $\mathrm{P}=0.84 ; \mathrm{FEV} 1 \%$ : $\mathrm{P}=0.80$ ). Likewise, the decrease in PEF was smaller in the IMT groups, but statistically significant difference was not detected $(\mathrm{P}=0.70)$ (Table 2).

The recovery of FIVC was a slightly better in the IMT compared to the PEP group between first and second postoperative day but without statistical significance $(\mathrm{P}=0.88)$. The drop at forced inspiratory volume in one second (FIV1) was smaller and recovery between the 
Table 3 Postoperative pulmonary function test values in proportion to preoperative value (mean ratio, with SD) in intervention groups

\begin{tabular}{|c|c|c|c|c|c|}
\hline Variable & \multicolumn{2}{|c|}{ PEP } & \multicolumn{2}{|c|}{ IMT } & $P$ value \\
\hline FVC & $0.69(0.32)$ & $0.72(0.20)$ & $0.72(0.18)$ & $0.80(0.15)$ & 0.38 \\
\hline FEV1 & $0.64(0.19)$ & $0.70(0.17)$ & $0.74(0.20)$ & $0.82(0.16)$ & 0.045 \\
\hline PEF & $0.61(0.20)$ & $0.67(0.19)$ & $0.69(0.21)$ & $0.80(0.17)$ & 0.051 \\
\hline FIVC & $0.67(0.19)$ & $0.73(0.15)$ & $0.74(0.17)$ & $0.82(0.17)$ & 0.098 \\
\hline FIV1 & $0.62(0.18)$ & $0.70(0.15)$ & $0.71(0.18)$ & $0.79(0.16)$ & 0.060 \\
\hline
\end{tabular}

$1 \mathrm{POD} /$ preop, first postoperative day value in proportion to pre-operative value; $2 \mathrm{POD} /$ preop, second postoperative day value in proportion to pre-operative value.

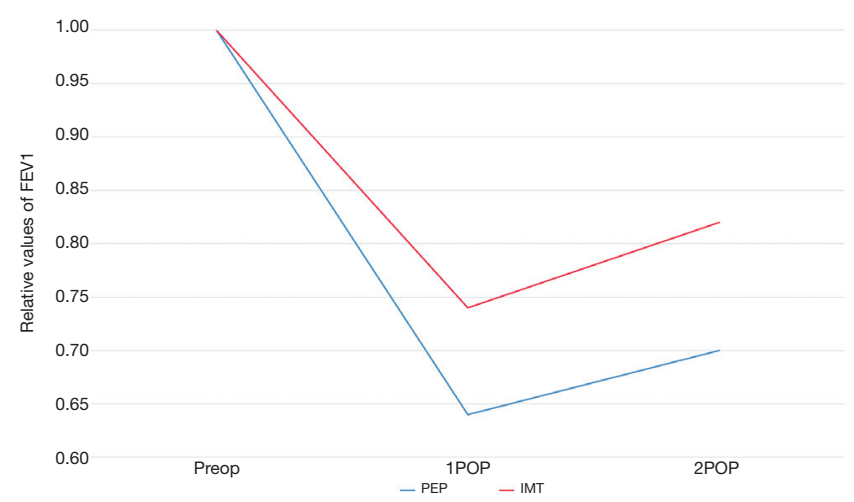

Figure 2 Relative values of FEV1 at different measurement dates in proportion to preoperative day value. Blue line illustrates water bottle PEP group and red line illustrates inspiratory muscle training (IMT) group.

first and the second postoperative day was better in the IMT group than in the water bottle PEP group, but the difference was not statistically significant $(\mathrm{P}=0.93)$ (Table 2$)$.

Maximal inspiratory pressure (MIP) was $84.55 \mathrm{cmH}_{2} \mathrm{O}$ $(S D=34.40)$ in the water bottle PEP group and $77.36 \mathrm{cmH}_{2} \mathrm{O}(\mathrm{SD}=26.74)$ in the IMT group preoperatively. The difference was not statistically significant. At the second postoperative day the maximal inspiratory pressure was $61.23 \mathrm{cmH}_{2} \mathrm{O}(\mathrm{SD}=23.92)$ in the water bottle PEP group and $60.77 \mathrm{cmH}_{2} \mathrm{O}(\mathrm{SD}=22.48)$ in the IMT group respectively. The decrease was more prominent in the water bottle PEP group but no statistically significant difference between the groups was noticed $(\mathrm{P}=0.40)$ (Table 2).

Pulmonary function values were also evaluated as relative values (calculated as a ratio of postoperative to preoperative) as presented at Table 3. Comparison was done by using two-

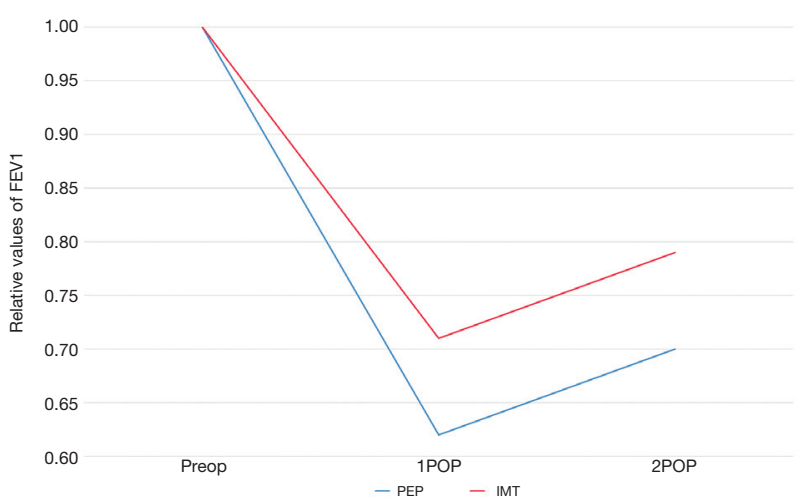

Figure 3 Relative values of FIV1 at different measurement dates in proportion to preoperative day value. Blue line illustrates water bottle PEP group and red line illustrates inspiratory muscle training (IMT) group.

way mixed factors repeated measures analysis of variances at three time points (preoperative, POD1 and POD2). The difference between intervention groups at relative values of FEV1 were increasing during first and second postoperative day, and the difference between intervention groups over the three time points was statistically significant $(\mathrm{P}=0.045)$, this being mainly in the difference at the second postoperative day, where the IMT group had statistically significantly higher relative FEV1 level as compared to PEP group $(\mathrm{P}=0.024)$. Also, $\mathrm{PEF}$ and FIV1 values seemed to be somewhat higher in the IMT compared to the PEP group, indicating that the decrease in pulmonary function values were smaller and the increase towards the preoperative value at POD2 was more rapid, but the differences were non-significant (PEF $\mathrm{P}=0.051$; FIV1 $\mathrm{P}=0.06$ ) (Table 3, Figures 2,3). 
Table 4 Patient-reported NRS-score for postoperative pain in intervention groups

\begin{tabular}{lccccc}
\hline \multirow{2}{*}{$\mathrm{NRS}^{\mathrm{a}}$} & \multicolumn{2}{c}{ 1POD $^{\mathrm{b}}$} & & \multicolumn{2}{c}{$2 \mathrm{POD}^{\mathrm{c}}$} \\
\cline { 2 - 5 } \cline { 4 - 5 } 1 & $\mathrm{PEP}$ & $\mathrm{IMT}$ & $\mathrm{PEP}$ & $\mathrm{IMT}$ \\
\hline 0 & 1 & 5 & 4 & 11 \\
2 & 0 & 6 & 7 & 2 \\
3 & 8 & 3 & 4 & 4 \\
4 & 2 & 5 & 2 & 2 \\
5 & 4 & 0 & 1 & 0 \\
6 & 4 & 1 & 3 & 2 \\
7 & 1 & 1 & 0 & 0 \\
8 & 2 & 0 & 1 & 0 \\
9 & 1 & 1 & 1 & 1 \\
10 & 0 & 0 & 0 & 0 \\
& 0 & 0 & 0 & 0 \\
\hline
\end{tabular}

NRS, numerical reference scale; 1POD, first postoperative day; 2POD, second postoperative day.

A significant difference was observed between intervention groups in experienced pain at POD1 $(\mathrm{P}=0.010)$. However, the difference became non-significant on POD2 $(\mathrm{P}=0.313)$ (Table 4). There was no major difference between the studied groups in the modalities of pain control and medications for pain.

The patient-reported average walking distances between first and second postoperative days were compared and values are presented in Table 5. Preoperative values were not recorded because admission times to the hospital varied too much to obtain uniform data. The mean walking distance at first postoperative day was 390 meters (SD =196 meters, range, 100-800 meters) in the PEP group and 397 meters ( $\mathrm{SD}=284$ meters, range, $0-900$ meters) in the IMT group. Corresponding values at POD2 were 720 meters ( $\mathrm{SD}=333$ meters, range, $140-1,450$ meters) in the PEP group and 721 meters ( $\mathrm{SD}=423$ meters, range, $2-1,500$ meters) in the IMT group. The differences were statistically nonsignificant $(\mathrm{P}=0.97)$.

Preoperative and postoperative chest X-rays (CXR's) were not available in all patients since the use of bed side ultrasound for evaluating pleural fluid or pneumothorax replaced routine postoperative CXR's in some cases, and in some patients thoracic CT scans were performed instead of CXR's preoperatively. Changes in the air-filled lung were measured for 17 patients in the water bottle PEP group and for 14 patients in the IMT group. There were no statistically significant differences between intervention groups when comparing changes in the air-filled lung or in the amount of atelectasis.

Postoperative pneumothorax was evaluated from CXR's for 20 of the water bottle PEP group patients and 18 of the IMT group patients. One patient in the IMT group had grade 1 pneumothorax and one patient in the water bottle PEP group had grade 2 pneumothorax, others had no pneumothorax as evaluated from the postoperative CXR's.

On the first postoperative day air leak occurred in $8.9 \%$ $(4 / 45)$ of all patients; two patients in the IMT group had minor $(0-200 \mathrm{~mL})$ air leak and in the water bottle PEP group one patient had minor and one patient moderate/ severe (over $200 \mathrm{~mL}$ ) air leak, respectively. On the second postoperative day, two patients in the PEP group and one patient in the IMT group had minor air leak, respectively. However, in water bottle PEP group increased air leak during physiotherapy necessitated pressure reduction in one patient at POD1 and POD2. In another patient in the PEP group physiotherapy was halted at the second postoperative day due to air leak. The differences in the occurrence of air leak and in the feasibility of physiotherapy between the study groups were statistically non-significant.

The mean hospital stay after surgery was 4.0 (SD $=2.8$ days $)$ in the PEP group and 3.3 days ( $\mathrm{SD}=1.7$ days) in the IMT group, but the difference was not significant. Chest tube drainage was continued for 2.4 days ( $\mathrm{SD}=2.7$ days) after surgery in the PEP group and 2.0 days ( $\mathrm{SD}=1.2$ days) in the IMT group. The difference was statistically non-significant.

\section{Discussion}

Our randomized controlled intervention study is one of the first to compare two different respiratory physiotherapy modalities, water bottle PEP and IMT physiotherapy, with patients undergoing minor lung resections.

The main finding of this comparison was that water bottle PEP and IMT physiotherapy were equal in efficiency when comparing spirometry values at three time points and walking distances at first and second postoperative day, with a significantly higher relative mean post-operative FEV1 value and less pain on POD1 in the IMT group.

The effect of thoracic surgery on lung function is multifactorial. Postoperative pain is a known limiting factor on breathing mechanics. Intercostal nerve pain can result 
Table 5 Mean postoperative walking distances of the intervention groups on the first and the second postoperative day

\begin{tabular}{lccccc}
\hline \multirow{2}{*}{ Variable } & \multicolumn{2}{c}{ PEP } & & \multicolumn{2}{c}{ IMT } \\
\cline { 2 - 4 } \cline { 5 - 6 } & 1POD & 2POD & 1POD & 2POD \\
\hline Walking distance, meters (SD) & $390.4(195.7)$ & $720.4(332.7)$ & $396.8(283.6)$ & $721.0(421.5)$ \\
Range, meters in 24 hours & $100-800$ & $140-1,450$ & $0-900$ & $2-1,500$ \\
\hline
\end{tabular}

1POD, first postoperative day; 2POD, second postoperative day.

from thoracoscopic access ports but VATS is now generally known to cause less trauma than thoracotomy, including muscle sparing procedures. Subsequently, postoperative recovery after VATS is faster (30). However, lung trauma from surgical manipulation and subsequent inflammation, as well as barotrauma to the contralateral lung from single lung ventilation can occur even after VATS. Apnea of the procedural sides' lung is usually required, and atelectatic areas may remain even after re-establishment of bilateral ventilation.

Breathing mechanics involve the diaphragm as an inspiratory muscle, as well as intercostal muscles, abdominal muscles, and accessory respiratory muscles such as the scalene and pectoral muscles. At rest, inspiration is an active process resulting mainly from diaphragmatic contraction and subsequent expansion of the lower thoracic cavity. Expiration results mainly from a passive contraction of the thoracic cavity. During exercise or other active respiratory effort, the other afore-mentioned muscle groups dynamically enhance breathing. However, non-synchronic action of the muscle groups can impair effective respiration (31).

We subjectively observed that IMT required a more conscious effort and more physical exertion from the patients. This can be an issue in debilitated patients, or those impeded by mental confusion or exhaustion caused by strong analgesics or an extensive surgical procedure. There were individual variations in pain medication in our series, but major thoracic surgical procedures were not included. A debilitating underlying condition such as metastatic cancer, old age, or co-morbidities can also affect the capability of the patient to co-operate. PEP exercises can be easier for the patients to perform, at least to some extent, because of the passive component of chest wall contraction as well as aid from abdominal, intercostal, and pectoral muscles. Conversely, IMT may be more effective as an exercise in patients who are able to perform it. A conscious effort required for the exercises will enhance co-ordination of all muscle groups involved in breathing to create a maximum inspiratory force against a pre-set resistance of the training device, in addition to overcoming the natural airway resistance.

Average reported daily walking distances were almost identical in both groups. This indicates a good similarity in the preoperative characteristics of the patients, but that a training period of one pre- and two postoperative days does not improve muscle strength or endurance. Instead, the beneficial effect to lung function is probably caused by immediate effect such as clearing of secretions, resolution of atelectasis and improved co-ordination of breathing mechanics. The same explanation may apply to why the inspiratory flow as measured by FIV1 was similar between groups.

We did not observe a difference in postoperative radiological manifestations of impaired lung expansion. The plain chest radiograph has limitations as a measure of lung dysfunction, and diffuse or limited areas of alveolar collapse or hypoventilation may appear unremarkable. These can be further overshadowed by an elevated diaphragm, poor inspiration, pleural plaques or fluid, chronic parenchymal lung disease, or differences in X-ray translucency such as caused by obesity. Also, only 17 of 45 patients had a chest $\mathrm{X}$-ray within 2 days from surgery, because of a policy to avoid imaging unless clinically indicated.

In PEP training, the expiratory force to overcome natural airway resistance as well as the pre-set $10 \mathrm{cmH}_{2} \mathrm{O}$ resistance of the water bottle device will also be directed to collapsed alveoli and atelectatic areas in the lungs. In IMT training, a negative pressure gradient is created during conscious inspiratory effort, diaphragmatic contraction and expansion of the lower parts of the thoracic cavity. This may promote a better expansion of basal atelectatic areas and help clear retained secretions. It may explain the significantly higher relative mean FEV1 value and a similar even though statistically non-significant trend in the other measured spirometry values for the IMT group.

We observed a significant pain advantage in the IMT group on day 1 , but not on day 2 . There was no difference in the frequency of thoracotomy between groups, nor the 
presence of a chest tube, or an epidural or sub-pleural catheter on either day, ruling out the typical explanations for a difference in pain. Also, majority of the patients did not have a pain treatment catheter and were only on peroral medication after surgery. Ropivacaine $2 \mathrm{mg} / \mathrm{mL}$ was routinely infiltrated into the access ports in patients not receiving a pain catheter, but its effect does not last till the next day. It is possible that the IMT group patients were more alert and determined on postoperative day two to perform the exercises despite of the required physical effort and pain experienced during training. Individual tolerance to pain medication, as well as limitations to the use of peroral opiates such as nausea and drowsiness and contraindications to anti-inflammatory analgesics may explain some of the differences. Discharge on day 3 or 4 was typical in our series, and our policy is to discontinue strong opiates before that. The NRS scale is a subjective measure of pain, so differences in reporting may also explain some differences. Furthermore, in both groups a pain level of over NRS value 5 was rarely reported, so that inability to train due to pain was uncommon.

Physiotherapy had to be modified due to air leak only in the two PEP group patients. While the difference was not statistically significant between groups, this is in accordance with our previous finding of a significant advantage for the feasibility of IMT in spite of air leak in lobectomy and more extended lung resections (27).

Our general evidence supporting postoperative respiratory physiotherapy appears consistent. The mean hospital stay was short at 3 to 4 days for both group, and there was no hospital mortality considering that $40 \%$ $(18 / 45)$ of the patients were at risk of complication due to preoperative malignant diagnosis and 29\% (13/45) had diagnosed chronic lung disease. A moderately or severely impaired respiratory function was noted preoperatively in $36 \%(16 / 45)$. In all patients, pulmonary function significantly worsened immediately after surgery thus increasing the preoperative risk for pulmonary complications even though all underwent a less than lobectomy lung resection. The training was feasible for all of the IMT group patients and did not increase costs of hospitalization. Pain or air leak did not impede training in either group. IMT training may provide an advantage for rehabilitation by improving the awareness and proprioception of the respiratory mechanics in patients. It's mechanism of action could give better lung expansion by creation of a strong negative pressure gradient and a surrounding vacuum-like effect on the lung during inspiration.

Our observations are in accord with previous studies (14,20-22), including our own previous study (27) on the advantages of IMT for lung resection patients. Some previous studies did not demonstrate any benefit from respiratory physiotherapy, incentive spirometry or IMT physiotherapy following pulmonary surgery (7,32-34). Deductions from the aforementioned studies are hampered by variability in interventional methods, the timing of surgery, and the duration of procedures (24).

The strength of this study was the prospective randomized setting where analyses were conducted according to the intention to treat principle. The same physiotherapist guided the patients' exercises in both intervention groups, so there should be no difference between the groups in the quality of guidance.

There are several limitations to this study. The power calculation indicated that both intervention groups fulfilled the minimum quota, and there were not dropouts or changes to the allocated groups. A main weakness is still the small number of patients. Although there was no statistically significant difference between the types of operations performed, more PEP group patients had thoracotomies than the IMT group patients which may have affected recovery. Another weakness is that blinding the study was not possible because the same physiotherapist guided all the exercises and also performed the volumetric spirometry.

We used a daily walking distance in 24 hours as one of our secondary outcomes instead of six minutes walking distance (6MWD). The research setup was done in close accordance with the standard treatment protocol of our unit in order to avoid additional costs and 6MWD would have required extra personnel to monitor the tests. Consistent preoperative data on daily walking distances could not be obtained because the times of hospital admission and subsequent recruitment into the study varied from morning to late afternoon. Patient-reported walking distances may be sensitive to bias due to differences in patients' basic activity, their willingness to do the exercises and variability in the recording of their walking distances. The more systematic 6MWD test could have been more comparable indicator of performance between intervention groups (35). Also, improvement in 6MWD has been associated with pulmonary rehabilitation of COPD and lung transplant patients (36).

Furthermore, long-term recovery could not be studied. 
Due to short average overall hospital stays, the changes in pulmonary functions could not be studied beyond two days.

\section{Conclusions}

Immediate postoperative decrease in respiratory function was significant even after minor pleuro-pulmonary operations and using VATS. Respiratory physiotherapy was feasible in all patients and appears justified to avoid PPCs. A significant improvement in postoperative relative FEV1 values may demonstrate an advantage for IMT as compared to PEP training even though it may be more demanding for the patient. The observed strenuousness of the IMT training emphasizes the need of individual guidance by a respiratory physiotherapist, as well as optimized postoperative pain control.

\section{Acknowledgments}

Funding: This work was supported by Tampere Tuberculosis Foundation and Pirkanmaa Cultural Foundation.

\section{Footnote}

Reporting Checklist: The authors have completed the CONSORT reporting checklist. Available at https://dx.doi. org/10.21037/jtd-21-473

Trial Protocol: Available at https://dx.doi.org/10.21037/jtd21-473

Data Sharing Statement: Available at https://dx.doi. org/10.21037/jtd-21-473

Peer Review File: Available at https://dx.doi.org/10.21037/ jtd-21-473

Conflicts of Interest: All authors have completed the ICMJE uniform disclosure form (available at https://dx.doi. org/10.21037/jtd-21-473). Dr. SIL reports receiving funding from Tampere Tuberculosis Foundation and Pirkanmaa Cultural Foundation. The other authors have no conflicts of interest to declare.

Ethical Statement: The authors are accountable for all aspects of the work in ensuring that questions related to the accuracy or integrity of any part of the work are appropriately investigated and resolved. The trial was conducted in accordance with the Declaration of Helsinki (as revised in 2013). The study protocol (R13037) was approved by the Ethics Committee of Pirkanmaa Hospital District, Tampere, Finland. Patients allocated to this study signed informed consent files.

Open Access Statement: This is an Open Access article distributed in accordance with the Creative Commons Attribution-NonCommercial-NoDerivs 4.0 International License (CC BY-NC-ND 4.0), which permits the noncommercial replication and distribution of the article with the strict proviso that no changes or edits are made and the original work is properly cited (including links to both the formal publication through the relevant DOI and the license). See: https://creativecommons.org/licenses/by-nc-nd/4.0/.

\section{References}

1. Falcoz PE, Conti M, Brouchet L, et al. The Thoracic Surgery Scoring System (Thoracoscore): risk model for in-hospital death in 15,183 patients requiring thoracic surgery. J Thorac Cardiovasc Surg 2007;133:325-32.

2. Birim O, Kappetein AP, Bogers AJ. Charlson comorbidity index as a predictor of long-term outcome after surgery for nonsmall cell lung cancer. Eur J Cardiothorac Surg 2005;28:759-62.

3. Ferguson MK, Durkin AE. A comparison of three scoring systems for predicting complications after major lung resection. Eur J Cardiothorac Surg 2003;23:35-42.

4. Hulzebos EH, Helders PJ, Favié NJ, et al. Preoperative intensive inspiratory muscle training to prevent postoperative pulmonary complications in high-risk patients undergoing CABG surgery: a randomized clinical trial. JAMA 2006;296:1851-7.

5. Duggan M, Kavanagh BP. Perioperative modifications of respiratory function. Best Pract Res Clin Anaesthesiol 2010;24:145-55.

6. Vagvolgyi A, Rozgonyi Z, Kerti M, et al. Effectiveness of pulmonary rehabilitation and correlations in between functional parameters, extent of thoracic surgery and severity of post-operative complications: randomized clinical trial. J Thorac Dis 2018;10:3519-31.

7. Sharafkhaneh A, Falk JA, Minai OA, et al. Overview of the perioperative management of lung volume reduction surgery patients. Proc Am Thorac Soc 2008;5:438-41.

8. Agostini P, Singh S. Incentive spirometry following thoracic surgery: what should we be doing? Physiotherapy 
2009;95:76-82.

9. Kaneda H, Saito Y, Okamoto M, et al. Early postoperative mobilization with walking at 4 hours after lobectomy in lung cancer patients. Gen Thorac Cardiovasc Surg 2007;55:493-8.

10. Sanchez-Lorente D, Navarro-Ripoll R, Guzman R, et al. Prehabilitation in thoracic surgery. J Thorac Dis 2018;10:S2593-600.

11. Weiner P, Man A, Weiner M, et al. The effect of incentive spirometry and inspiratory muscle training on pulmonary function after lung resection. J Thorac Cardiovasc Surg 1997;113:552-7.

12. Nici L. Preoperative and postoperative pulmonary rehabilitation in lung cancer patients. Thorac Surg Clin 2008;18:39-43.

13. Huang J, Lai Y, Zhou X, et al. Short-term high-intensity rehabilitation in radically treated lung cancer: a threearmed randomized controlled trial. J Thorac Dis 2017;9:1919-29.

14. Zhou K, Su J, Lai Y, et al. Short-term inpatient-based high-intensive pulmonary rehabilitation for lung cancer patients: is it feasible and effective? J Thorac Dis 2017;9:4486-93.

15. Templeton R, Greenhalgh D. Preoperative rehabilitation for thoracic surgery. Curr Opin Anaesthesiol 2019;32:23-8.

16. Steffens D, Beckenkamp PR, Hancock M, et al. Preoperative exercise halves the postoperative complication rate in patients with lung cancer: a systematic review of the effect of exercise on complications, length of stay and quality of life in patients with cancer. Br J Sports Med 2018;52:344.

17. Varela G, Novoa NM, Agostini P, et al. Chest physiotherapy in lung resection patients: state of the art. Semin Thorac Cardiovasc Surg 2011;23:297-306.

18. Freynet A, Falcoz PE. Does non-invasive ventilation associated with chest physiotherapy improve outcome after lung resection? Interact Cardiovasc Thorac Surg 2008;7:1152-4.

19. Fujimoto S, Nakayama T. Effect of combination of preand postoperative pulmonary rehabilitation on onset of postoperative pneumonia: a retrospective cohort study based on data from the diagnosis procedure combination database in Japan. Int J Clin Oncol 2019;24:211-21.

20. Brunelli A, Charloux A, Bolliger CT, et al. ERS/ESTS clinical guidelines on fitness for radical therapy in lung cancer patients (surgery and chemo-radiotherapy). Eur Respir J 2009;34:17-41.
21. Kendall F, Abreu P, Pinho P, et al. The role of physiotherapy in patients undergoing pulmonary surgery for lung cancer. A literature review. Rev Port Pneumol (2006) 2017;23:343-51.

22. Kendall F, Oliveira J, Peleteiro B, et al. Inspiratory muscle training is effective to reduce postoperative pulmonary complications and length of hospital stay: a systematic review and meta-analysis. Disabil Rehabil 2018;40:864-82.

23. Morano MT, Araújo AS, Nascimento FB, et al. Preoperative pulmonary rehabilitation versus chest physical therapy in patients undergoing lung cancer resection: a pilot randomized controlled trial. Arch Phys Med Rehabil 2013;94:53-8.

24. Ge X, Wang W, Hou L, et al. Inspiratory muscle training is associated with decreased postoperative pulmonary complications: Evidence from randomized trials. J Thorac Cardiovasc Surg 2018;156:1290-1300.e5.

25. Pehlivan E, Mutluay F, Balcı A, et al. The effects of inspiratory muscle training on exercise capacity, dyspnea and respiratory functions in lung transplantation candidates: a randomized controlled trial. Clin Rehabil 2018;32:1328-39.

26. Gosselink R, De Vos J, van den Heuvel SP, et al. Impact of inspiratory muscle training in patients with COPD: what is the evidence? Eur Respir J 2011;37:416-25.

27. Lähteenmäki S, Sioris T, Mahrberg H, et al. Inspiratory training and immediate lung recovery after resective pulmonary surgery: a randomized clinical trial. J Thorac Dis 2020;12:6701-11.

28. Durheim MT, Kim S, Gulack BC, et al. Mortality and Respiratory Failure After Thoracoscopic Lung Biopsy for Interstitial Lung Disease. Ann Thorac Surg 2017;104:465-70.

29. Kainu A, Lindqvist A, Sovijärvi AR. Prevalence of abnormal findings when adopting new national and international Global Lung Function Initiative reference values for spirometry in the Finnish general population. Eur Clin Respir J 2016;3:30658.

30. Park TY, Park YS. Long-term respiratory function recovery in patients with stage I lung cancer receiving video-assisted thoracic surgery versus thoracotomy. J Thorac Dis 2016;8:161-8.

31. Aliverti A. The respiratory muscles during exercise. Breathe (Sheff) 2016;12:165-8.

32. Nellessen A, Hernandes NA, Pitta F. Physiotherapy and rehabilitative interventions in patients with chronic respiratory diseases: exercise and non-exercise treatment. Panminerva Med 2013;55:197-209. 
33. Ries AL, Bauldoff GS, Carlin BW, et al. Pulmonary Rehabilitation: Joint ACCP/AACVPR Evidence-Based Clinical Practice Guidelines. Chest 2007;131:4S-42S.

34. Freitas ER, Soares BG, Cardoso JR, et al. Incentive spirometry for preventing pulmonary complications after coronary artery bypass graft. Cochrane Database Syst Rev
2012;(9):CD004466.

35. Erratum: ATS Statement: Guidelines for the Six-Minute Walk Test. Am J Respir Crit Care Med 2016;193:1185.

36. Rasekaba T, Lee AL, Naughton MT, et al. The six-minute walk test: a useful metric for the cardiopulmonary patient. Intern Med J 2009;39:495-501.

Cite this article as: Lähteenmäki SI, Sioris T, Mahrberg HSS, Rinta-Kiikka IC, Laurikka JO. A randomized trial comparing inspiratory training and positive pressure training in immediate lung recovery after minor pleuro-pulmonary surgery. J Thorac Dis 2021;13(8):4690-4702. doi: 10.21037/jtd-21-473 